\title{
Exploring ultrasound-modulated optical tomography at clinically useful depths using the photorefractive effect
}

Puxiang Lai, Yuta Suzuki, Xiao Xu, Lihong V. Wang

Puxiang Lai, Yuta Suzuki, Xiao Xu, Lihong V. Wang, "Exploring ultrasoundmodulated optical tomography at clinically useful depths using the photorefractive effect," Proc. SPIE 8581, Photons Plus Ultrasound: Imaging and Sensing 2013, 85812X (4 March 2013); doi: 10.1117/12.2003270

SPIE. Event: SPIE BiOS, 2013, San Francisco, California, United States 


\title{
Exploring Ultrasound-Modulated Optical Tomography at Clinically Useful Depths Using the Photorefractive Effect
}

\author{
Puxiang Lai, Yuta Suzuki, Xiao Xu, and Lihong V. Wang \\ Washington University in St. Louis \\ Department of Biomedical Engineering, Optical Imaging Laboratory \\ Campus Box 1097, 1 Brookings Drive, St. Louis, Missouri 63130, USA
}

\begin{abstract}
For years, ultrasound-modulated optical tomography (UOT) has been proposed to image optical contrasts deep inside turbid media (such as biological tissue) at an ultrasonic spatial resolution. The reported imaging depth so far, however, has been limited, preventing this technique from finding broader applications. In this work, we present our latest experimental explorations that push UOT to clinically useful imaging depths, achieved through optimizing from different aspects. One improvement is the use of a large aperture fiber bundle, which more effectively collects the diffused light, including both ultrasound-modulated and unmodulated portions, from the turbid sample and then sends it to the photorefractive material. Another endeavor is employment of a large aperture photorefractive polymer film for demodulating the ultrasound-induced phase modulation. Compared with most UOT detection schemes, the polymer film based setup provides a much higher etendue as well as photorefractive two-beam-coupling gain. Experimentally, we have demonstrated enhanced sensitivity and have imaged through tissue-mimicking samples up to $9.4 \mathrm{~cm}$ thick at the ultrasonically-determined spatial resolutions.
\end{abstract}

Keywords: optical imaging, ultrasound modulation, ultrasound-modulated optical tomography, acousto-optic imaging, photorefractive crystal, photorefractive polymer, tissue-mimicking phantom, biological tissue, optical fiber bundle

\section{INTRODUCTION}

As a non-invasive, non-ionizing, and relatively cost-effective technique that is able to provide functional information and to distinguish different tissue types, optical imaging plays a more and more important role in biomedical diagnosis [1]. Its wide applications, however, are fundamentally hindered by the fact that light is highly diffused in biological tissue, causing a trade-off between the imaging depth and the resultant spatial resolution. To overcome this limitation of reduced spatial resolution due to strong light diffusivity when using optical imaging alone, researchers have proposed different ways to combine optical sensing with another imaging modality, e.g., ultrasound, taking advantage of the contrast provided by the pure optics and the spatial resolution of the ultrasound [2]. Ultrasound-modulated optical tomography (UOT), also called acousto-optic imaging, is such an example [3, 4].

Further author information: Puxiang Lai, plai@wustl.edu, 1-314-935 9587

Lihong V. Wang, lhwang@wustl.edu, 1-314- 9356152

Photons Plus Ultrasound: Imaging and Sensing 2013, edited by Alexander A. Oraevsky, Lihong V. Wang, Proc. of SPIE Vol. 8581, 85812X - (C) 2013 SPIE · CCC code: 1605-7422/13/\$18 - doi: 10.1117/12.2003270 
In this technique, a focused ultrasound field is applied to modulate the propagation of light inside a turbid medium. Photons are multiply diffused, which leads to the formation of random speckles outside the sample. Photons traversing the ultrasound focal region, however, experience an additional frequency shift that is equal to the ultrasound frequency, through the displacements of scatterers and the variation in the medium's index of refraction [5, 6]. Since such modulation only occurs within the acousto-optic interaction volume, and the depth of the modulation is intrinsically related to local optical properties, detecting ultrasound-modulated photons yields optically relevant information at the ultrasonic resolution. Thus far, UOT has seen many applications, such as conventional optical contrast imaging [7], multi-wavelength functional imaging [8], mechanical contrast imaging [9, 10], fluorescence imaging [11], quantitative measurement of optical properties [12], real-time monitoring of thermal necrosis [13], etc. Most of these studies, however, were performed in tissue-mimicking phantoms or ex-vivo tissue samples with a limited depth less than the clinically useful thickness (usually $5-10 \mathrm{~cm} \mathrm{[14]).} \mathrm{The} \mathrm{depth} \mathrm{insufficiency} \mathrm{is} \mathrm{partially} \mathrm{caused} \mathrm{by} \mathrm{the} \mathrm{fact} \mathrm{that} \mathrm{the} \mathrm{number}$ of modulated photons is much less compared with the number of un-modulated photons, posing a strict requirement to extract a very small amount (typically $0.1-1 \%$ of the total power) of MHz-ordered frequency-shifted modulated photons from a much stronger untagged background that is on the order of THz [15]. Moreover, since photons travel along different optical paths inside the turbid medium, speckles forming on the sample surface have random phase differences. Therefore, looking at one or only a few speckles results in a low flux of tagged-light. While directly detecting multiple speckles onto a single-element detector does not coherently sum up the amount of tagged photons, it apparently increases useless untagged background levels. Different methods have been proposed to overcome this dilemma and enhance the signal detection sensitivity. These efforts include, but are not limited to, CCD-based parallel detection [16, 17], photorefractive crystal (PRC)-based interferometry [18, 19], Fabry-Perot interferometry [20, 21], as well as spectral hole burning-based filtering methods [22, 23]. In this work, we present our latest explorations [24, 25] to push UOT to clinically useful thicknesses by improving the etendue of diffused light collection and the efficiency of de-modulation of a photorefractive interferometric UOT system.

\section{METHODOLOGY AND MATERIALS}

Fig. 1 shows the experimental setup used in the study. The detailed descriptions of this setup can be found in literatures $[24,25]$, and thus are not iterated in this report. Several things, however, need to be specially described.

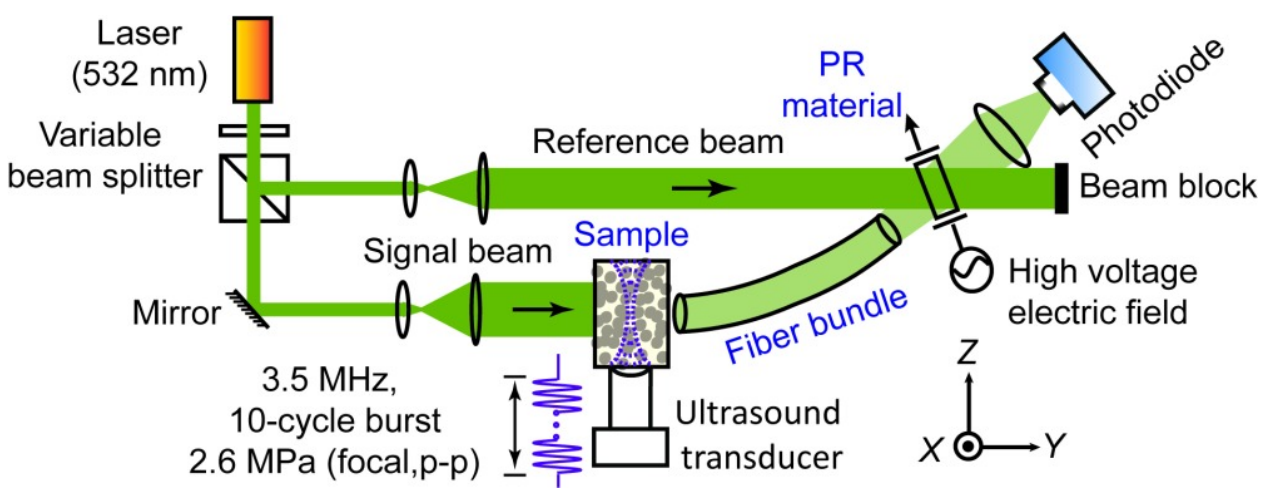

Fig. 1. System experimental setup. $Y$ is the optical illumination direction, and $Z$ is the ultrasound propagation direction. 
First, the diffused light from the experimental sample, including both ultrasound-modulated and unmodulated portions, was collected using an optical fiber bundle from Edmund Optics (NT38-659). As illustrated in Fig. 2, multiple optical fibers were tightly packed into the round bundle, which had a net clear aperture of $12.7 \mathrm{~mm}$, a length of $305 \mathrm{~mm}$, a high NA of 0.55 , and a full acceptance angle of $68^{\circ}$. This fiber bundle offered improved light throughput (or etendue) compared with standard single fiber assemblies, and greater flexibility/convenience compared with regular lenses used in free space.

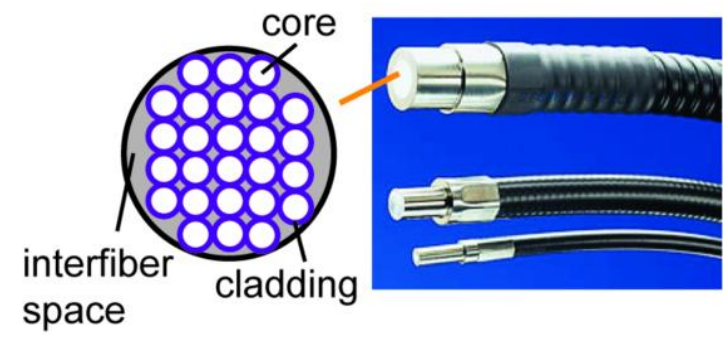

Fig. 2 Illustration of the optical fiber bundle used in this study

Second, two types of photorefractive materials, as shown in Fig. 3, were used in this study to efficiently convert the ultrasound-induced frequency and phase modulation into an intensity modulation that could be detected by a photodiode $[18,19,26]$. The first type was a $\mathrm{Bi}_{12} \mathrm{SiO}_{20}$ (BSO) photorefractive crystal from Elan, Russia that had a dimension of $10 \times 10 \times 5 \mathrm{~mm}^{3}$ along the $X, Z, Y$ directions. Under our experimental conditions (described later), the crystal yielded a response speed of $\sim 100 \mathrm{~ms}$, and two-wave-mixing (TWM) gain coefficients of 0.81 and $0.26 \mathrm{~cm}^{-1}$, respectively, with and without a $2.1 \mathrm{kHz}, 8 \mathrm{kV} / \mathrm{cm}$ high voltage AC electronic field applied across the crystal (along the $X$ direction). The second type is a photorefractive polymer (PRP) film from Nitto Denko Technical, CA, USA. The PRP, $50.8 \times 50.8 \times 0.1$ $\mathrm{mm}^{3}$ along $X, Z$, and $Y$, respectively, had much larger dimensions along the $X$ and $Z$ directions than the BSO, as shown in Fig. 3. In our study, a 400-1000 kV/cm high voltage DC electronic field was applied along the PRP (along the $Y$ direction) to enhance the TWM gain coefficient up to $9.2 \mathrm{~cm}^{-1}$. However, it must be noted that this polymer film was relatively slow, with a response time up to several seconds under our experimental conditions, which is not desirable for future in-vivo applications to compensate the speckle decorrelation caused by the physiological motions. However, to demonstrate deep imaging in a non in-vivo scenario, this slow response speed was practically adequate, as will be shown later in this report.

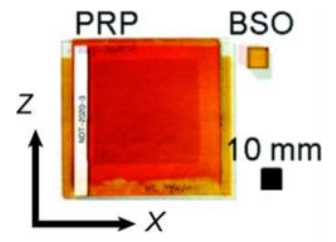

Fig. 3 Dimension comparison of the BSO photorefractive crystal and the photorefractive polymer (PRP) film

At this point, it is necessary to highlight the advantage of using this large aperture PRP in terms of diffused light collection etendue. Etendue is an important parameter used to specify the geometric capability of an optical system to 
transmit, and/or receive radiation (in our case, light), its throughput. Numerically, it is defined as $G=\pi A \sin ^{2}(\Omega / 2)$, where $A$ is the active surface area of the optical component, and $\Omega$ represents the emission/acceptance angle. In UOT, the experimental samples usually have apertures or diameters of several centimeters, and accordingly large output etendues of 5,000-30,000 $\mathrm{mm}^{2} \mathrm{sr}$, considering an $\Omega$ of $180^{\circ}$ as light gets multiply scattered within the experimental sample. As shown in Fig. 4, previous detections schemes that allowed coherent signal summation over multiple speckles, including confocal Fabry-Perot interferometers (CFPI), PRC-based interferometers, and spectral hole-burning crystals, had much smaller etendues due to their limited dimensions and/or acceptance angles. It meant only a small portion of tagged photons were effectively used and contributed to the final UOT signal levels. This, accompanied with the factors discussed earlier in the Introduction, makes it challenging to image through thick turbid media within the illumination safety limits for sound [27] and light [28]. Comparably, the PRP film used in this study provided an etendue up to 400-500 $\mathrm{mm}^{2} \mathrm{sr}$, imperfect yet much larger than previous schemes, promising a manifold increase in the UOT signal-to-noise ratio due to the increase of useful tagged photons.

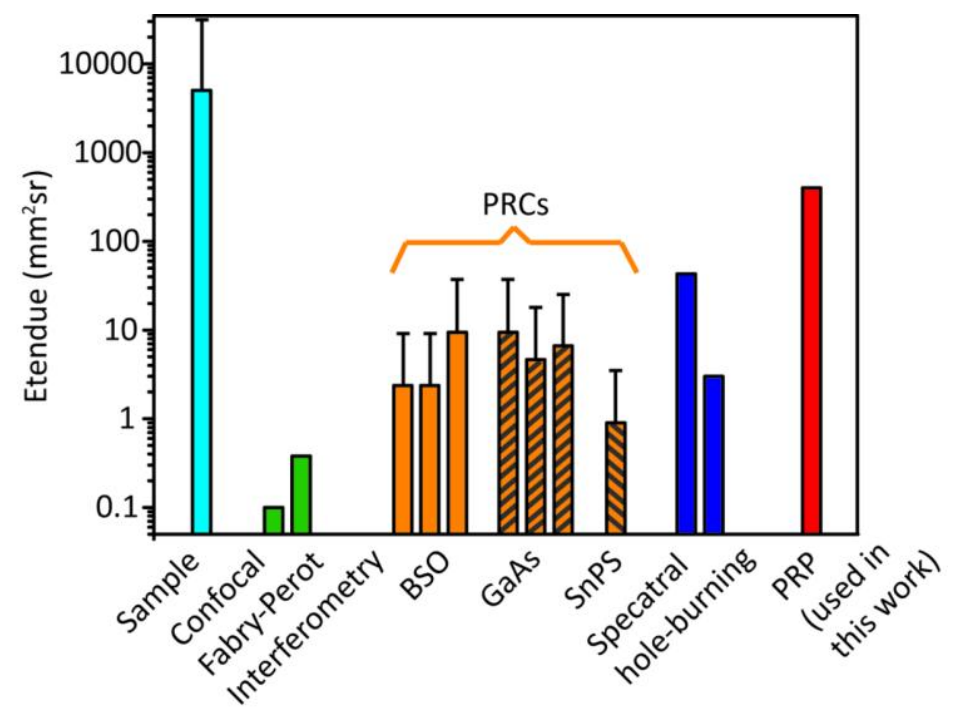

Fig. 4 Comparison of etendue for experiment sample and different detection schemes. Each column represents one case reported in literatures: confocal Fabry-Perot interferometers (CFPI) [20, 21], BSO crystals [9, 18, 24], GaAs crystals [12, 19, 29], SnPS crystals [30], spectral hole-burning crystals [22, 23], and the PRP film used in this work. The error bar of the experimental sample comes from the variance of sample surface dimensions (ranging from $4 \times 4$ to $10 \times 10 \mathrm{~cm}^{2}$ ), while the error bars for the PRCs are from the approximation of $\Omega$ (20-40 according to Ref. [23]).

In this study, two phantoms that mimicked optical properties of tissue were used as the experimental samples. These phantoms were $6 \mathrm{~cm}$ and $9.4 \mathrm{~cm}$ thick, respectively. Both were composed of 10\% (by weight) porcine gelatin (Sigma-Aldrich, MO, USA), 89\% water, and 1\% Intralipid (diluted from 20\% Intralipid from Fresenius Kabi, Germany). The resultant $\mu_{s}^{\prime}=10 \mathrm{~cm}^{-1}$, measured by oblique-incidence reflectometry [31], and the absorption coefficient $\mu_{\mathrm{a}}$ was $0.12 \mathrm{~cm}^{-1}$, measured by a spectrophotometer (Cary 50, Varian, CA, USA). To provide optical contrasts inside these two phantoms, absorbing objects were embedded at the central plane of the samples. The absorbers were made from the same 
material with the background but dyed with India ink to have a higher absorption coefficient at $2 \mathrm{~cm}^{-1}$. More details about these samples and how the absorbers were deployed will be given later.

Fig. 5 shows two examples of normalized UOT signal waveform when the ultrasound burst from a $3.5 \mathrm{MHz}$ transducer (Panametrics A318S, Olympus NDT, MA, USA) propagated outside and across an optical absorber that was embedded inside the 6-cm thick phantom. As seen, when the ultrasound burst travelled away from the absorber (the green curve), the modulated-light power increased with the in situ pressure amplitude, forming a summit around 38-39 $\mu$ s when the burst arrived at the ultrasound focal position ( $38.1 \mathrm{~mm}$ away from the transducer surface). With the presence of the absorber, however, the modulated-light power began to reduce when the ultrasound burst encountered the absorbing region, due to the decreased amount of modulated photons and the efficiency of ultrasound modulation [6, 12, 32]. Therefore, the valley of the UOT signal is seen as the signature of the optical absorber.

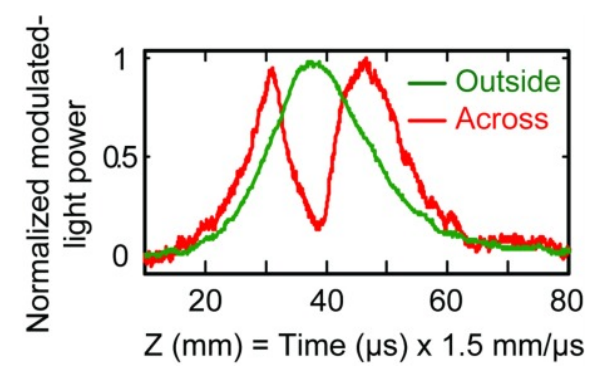

Fig. 5 Examples of UOT signal waveform with ultrasound pulses propagating outside (green curve) and across (red curve) an optical absorber embedded inside the 6-cm thick phantom.

\section{RESULTS AND DISCUSSION}

Fig. 6(a) shows the cross-section photograph of the 6-cm thick tissue-mimicking phantom mentioned earlier. There were three absorbing objects that had a higher optical absorption coefficient $\left(2 \mathrm{~cm}^{-1}\right)$ than the background. This cross-section was embedded at $Y=3 \mathrm{~cm}$ in the $6-\mathrm{cm}$ thick sample. Before the experiment, the ultrasound focus was aligned to intersect with the needle on the left in both $Y$ and $Z$ directions. During the experiment, the BSO photorefractive crystal was used in the detection scheme [24]. And a signal beam of $35 \mathrm{~mm}$ diameter, $208 \mathrm{~mW} / \mathrm{cm}^{2}$, a reference beam of $10 \mathrm{~mm}$ diameter, $30 \mathrm{~mW} / \mathrm{cm}^{2}$, and a 10 -cycle burst with a $2.6 \mathrm{MPa}$ (peak-to-peak) focal pressure, and a repetition rate of $100 \mathrm{~Hz}$, were employed. Two groups of measurements were performed. First, the light and sound components remained stationary, but the sample scanned along the $X$ direction at a step size of $0.3175 \mathrm{~mm}$. In the second group of measurements, the light and the sample kept still, but the ultrasound focus was scanned along the $Y$ direction with the sample fixed at the position of $X=6.35 \mathrm{~mm}$. At each position, one UOT scan line ("A line") as shown in Fig. 5 was obtained, averaging over 64 ultrasound bursts. Fig. 6(b) and (c) show the resulting 2-D UOT images, with each scan line normalized to its peak power for better visualization contrast. Note the distance along the $Z$ direction was converted from the product of temporal position with the sound speed in the phantom $(\sim 1.5 \mathrm{~mm} / \mu \mathrm{s})$. In Fig. $6(\mathrm{~b})$, the three embedded absorbers can be clearly seen in the $X Z$ plane, even though Obj3 was only partially imaged due to the limited scanning range of our translation stages. In Fig. 6(c), Obj1 was also clearly revealed in the $Y Z$ plane. Moreover, the profiles (Fig. 6 d-f) along the dashed lines in Fig. 6(b) and (c) provided more information, like the dimensions of the objects, the interspacing 
between the objects, and the resolutions along the $X$ and $Z$ directions. For example, in Fig. 6(d), Obj1 was centered at $X \approx$ $6.0 \mathrm{~mm}, \sim 2.4 \mathrm{~mm}$ wide (all imaged sizes in this study were determined from the full-width at half maximum of the contrasts), and $\mathrm{Obj} 2$ at $X \approx 16.9 \mathrm{~mm}, \sim 4.3 \mathrm{~mm}$ wide. These were quite consistent with what can be seen from the cross-section photograph (Fig. 6a) that $\mathrm{Ob} 1$ was $\sim 2.1 \mathrm{~mm}$, Obj2 was $\sim 4.0 \mathrm{~mm}$, and their center-to-center distance was $\sim 10 \mathrm{~mm}$. The resolution of UOT along the $X$ direction, estimated from the 1/4-3/4 of the imaging contrasts, was $\sim 1.05$ $\mathrm{mm}$, which was only slightly wider than the ultrasound focal width $(0.875 \mathrm{~mm})$. In Fig. 6(e), Obj1 was about $5.1 \mathrm{~mm}$ long, and was, again, reasonably consistent with its actual length of $4.5 \mathrm{~mm}$ shown in the photograph. The imaging resolution along the $Z$ direction was $\sim 2.4 \mathrm{~mm}$, which is $0.5-0.6$ of the ultrasound burst length (10 cycles of $3.5 \mathrm{MHz}$ is about $4.3 \mathrm{~mm}$ long spatially). Lastly, Obj1 was $\sim 3.8 \mathrm{~mm}$ wide from the profile shown in Fig. 6(f), a value very close to its actual dimension $(3.5 \mathrm{~mm})$. The estimated imaging resolution in the $Y$ direction was $\sim 1.17 \mathrm{~mm}$, and was close to the focal width of the ultrasound field.

(a)

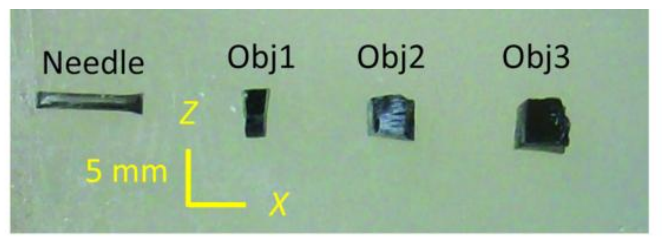

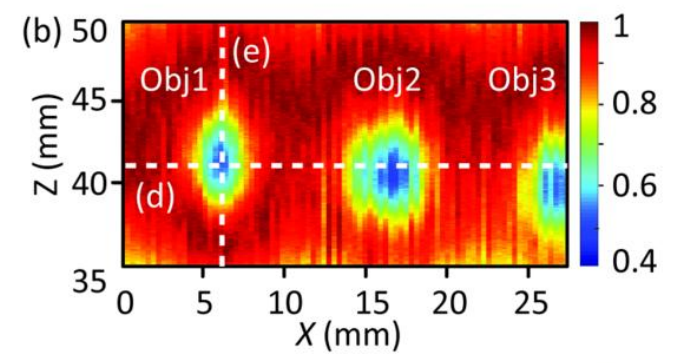

(d)

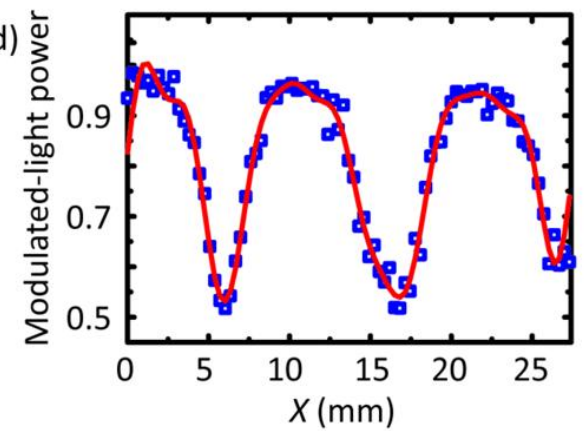

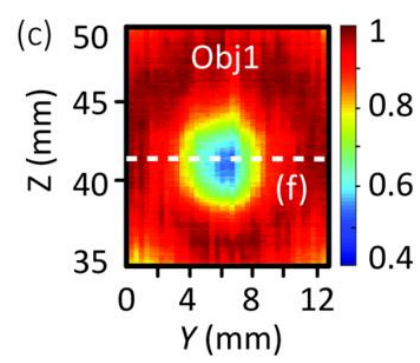

(e)

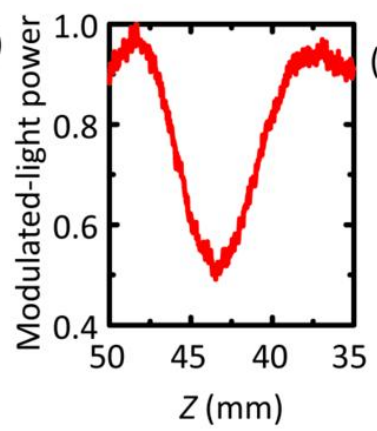

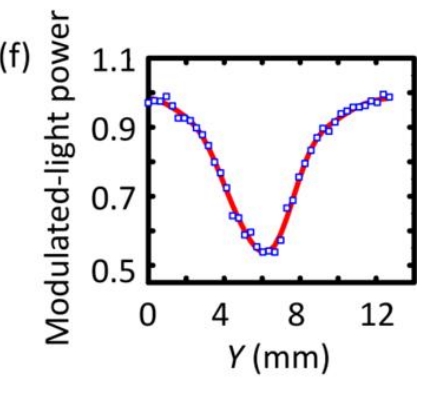

Fig. 6 (a) The cross-section photograph of the 6-cm thick phantom. (b) The resultant 2-D UOT image of the phantom in the $X Z$ plane with $Y=3 \mathrm{~cm}$. (c) The 2-D UOT image in a $Y Z$ plane across Obj1 ( $X=6.35 \mathrm{~mm}$ in Fig. 6b). (d-f) Modulated light power distributions along dashed lines labeled in Figs. (b) and (c), respectively. In (d) and (f), the blue squares are the measured data, and the red curves the FFT smoothed results. Reproduced from Ref. [24].

To image through the 9.4-cm thick phantom sample (its cross-section shown in Fig. 7a), the photorefractive polymer film discussed earlier was used to enhance the detection sensitivity without the need of employing much more intense light and sound illuminations [25]. Except for the replacement of the photorefractive material, other experiment parameters 
remained very similar or unchanged from the last measurements performed for Fig. 6. For example, the used signal beam had a diameter of $24 \mathrm{~mm}$, an intensity of $192 \mathrm{~mW} / \mathrm{cm}^{2}$, and the reference beam a diameter of $30 \mathrm{~mm}$, an intensity of 9.9 $\mathrm{mW} / \mathrm{cm}^{2}$. The ultrasound field, the averaging times of UOT signal, the way of scanning the experimental sample, and the signal processing methods were kept identical to those used for Fig. 6. The resulting "B-Mode" 2-D UOT image of the cross-section was shown in Fig. 7(b), from which one can tell the existence, dimensions, inter-spacing of the two embedded absorbers, and the resolutions in the $X Z$ plane. Note that this UOT image was obtained with a relatively straightforward and inexpensive apparatus in a tissue-mimicking phantom sample with a thickness of $9.4 \mathrm{~cm}$, which is equivalent to 94 transport mean free paths, or $9.4 \mathrm{~cm}$ thickness of normal human breast tissue, a clinically useful depth that had never been achieved, not to mention explored, until most recently [23-25].

(a)

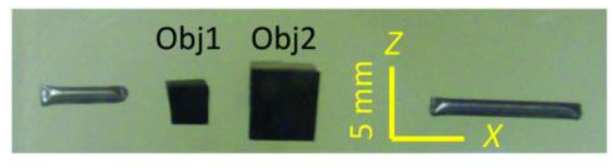

(b)

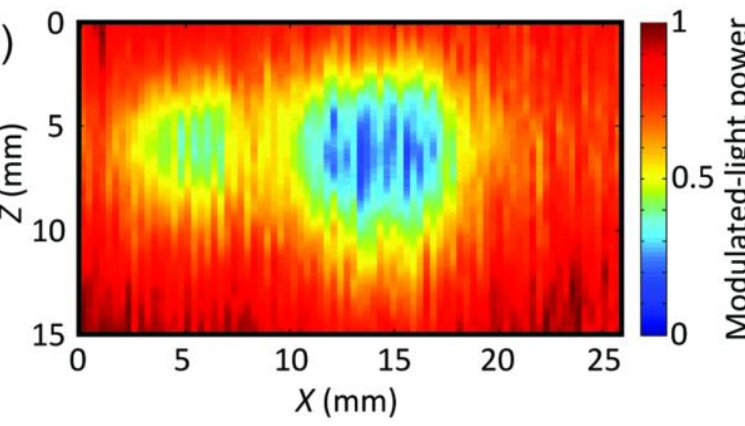

Fig. 7 (a) Cross-section of the $9.4 \mathrm{~cm}$-thick phantom sample, embedded with two absorbing objects at its central $Y$ plane $(Y=4.7 \mathrm{~cm})$. (b) The resulting 2-D UOT image of the central cross-section. Reproduced from Ref. [25].

\section{SUMMARY AND FUTURE WORK}

In summary, we reported our latest improvements to a relatively simple and cost-effective photorefractive-based UOT system. Our efforts included the use of a large aperture optical fiber bundle to improve the diffused light collection convenience and efficiency, and a photorefractive polymer to improve the etendue and two-wave-mixing gain coefficient for coherent de-modulation of ultrasound-induced frequency/phase modulation. Experimentally, we demonstrated UOT imaging at clinically useful thicknesses up to $9.4 \mathrm{~cm}$ in tissue-mimicking phantom samples, within the light [28] and sound [27] safety limits. That being said, some more work needs to done to make our apparatus towards clinical applications. For example, the system needs to be modified to work at a more tissue-friendly optical wavelength such as 700-800 nm or $1064 \mathrm{~nm}$ for deeper penetration depth in tissue, benefitting from less optical tissue attenuation and higher ANSI safety limits [28]. Moreover, faster responding photorefractive materials (e.g. GaAs crystal [33], SnPS crystal [30], and fast polymers [34]) are desired to better compensate the in vivo speckle decorrelation (1-10 kHz) from physiological motions.

\section{ACKNOWLEDGEMENTS}

The authors thank Sandra Matteucci for editing the manuscript, and Nitto Denko Technical at Oceanside, CA for providing the photorefractive polymer film used in the current study. This research is sponsored in part by the National Academies Keck Futures Initiative grant IS 13, National Institute of Health grants DP1 EB016986 (NIH Director's Pioneer Award), R01 EB000712 and U54 CA136398. L.W. has a financial interest in Microphotoacoustics, Inc. and Endra, Inc., which, however, did not support this work. 


\section{REFERENCES}

[1] L. V. Wang, and H. Wu, [Biomedical Optics: Principles and Imaging] John Wiley and Sons, Hoboken, New Jersey(2007).

[2] L. V. Wang, "Ultrasound-mediated biophotonic imaging: A review of acousto-optical tomography and photo-acoustic tomography,” Disease Markers, 19, 123-138 (2004).

[3] D. S. Elson, R. Li, C. Dunsby et al., "Ultrasound-mediated optical tomography: a review of current methods," Interface Focus, 1, 632-648 (2011).

[4] S. G. Resink, W. Steenbergen, and A. C. Boccara, "State-of-the art of acoust-optic sensing and imaging of turbid media," Journal of Biomedical Optics, 17(4), 040901 (2012).

[5] L. V. Wang, "Mechanisms of ultrasound modulation of multiply scattered coherent light: An analytic model," Phys. Rev. Lett., 87(4), 043903 (2001).

[6] S. Sakadzic, and L. V. Wang, "Modulation of multiply scattered coherent light by ultrasound pulses: An analytical model,” Phys. Rev. E, 72(033620), 033620 (2005).

[7] S. Farahi, E. Benoit, A. A. Grabar et al., "Time resolved three-dimensional acousto-optic imaging of thick scattering media," Opt. Lett., 37(13), 2754-2756 (2012).

[8] C. Kim, and L. V. Wang, "Multi-optical-wavelength ultrasound-modulated optical tomography: a phantom study," Opt. Lett., 32(16), 2285-2287 (2007).

[9] X. Xu, H. Zhang, P. Hemmer et al., "Photorefractive detection of tissue optical and mechanical properties by ultrasound modulated optical tomography,” Opt. Lett., 32(6), 656-658 (2007).

[10] R. Li, D. S. Elson, C. Dunsby et al., "Effects of acoustic radiation force and shear waves for absorption and stiffness sensing in ultrasound modulated optical tomography,” Opt. Express, 19(8), 7299-7311 (2011).

[11] B. Yuan, S. Uchiyama, Y. Liu et al., "High-resolution imaging in a deep turbid medium based on an ultrasound-switchable fluorescence technique,” Applied Physics Letters, 101(3), 033703-5 (2012).

[12] P. Lai, R. A. Roy, and T. W. Murray, "Quantitative characterization of turbid medium using pressure contrast acousto-optic imaging," Opt. Lett., 34(18), 2850-2852 (2009).

[13] P. Lai, J. R. McLaughlan, A. B. Draudt et al., "Real time monitoring of high-intensity focused ultrasound lesion formation using acousto-optic sensing," Ultrasound in Medicine and Biology, 37(2), 239-252 (2011).

[14] L. V. Wang, "Ultrasonic modulation of scattered in turbid media and a potential novel tomography in biomedicine," Photochem. Photobiol., 67(1), $41-49$ (1998).

[15] T. W. Murray, and R. A. Roy, "Illuminating Sound: Imaging Tissue Optical Properties with Ultrasound," Acoustics Today, 3(3), 17-23 (2007).

[16] S. Leveqe-Fort, A. C. Boccara, M. Lebec et al., "Ultrasonic tagging of photon paths in scattering media: parallel speckle modulation processing,” Opt. Lett., 24(3), 181-183 (1999).

[17] G. Yao, and L. V. Wang, "Theoretical and experimental studies of ultrasound-modulated optical tomography in biological tissue,” Appl. Opt., 39(4), 659-664 (2000).

[18] T. W. Murray, L. Sui, G. Maguluri et al., "Detection of ultrasound-modulated photons in diffuse media using the photorefractive effect,” Opt. Lett., 29(21), 2509-2511 (2004).

[19] F. Ramaz, B. C. Forget, M. Atlan et al., "Photorefractive detection of tagged photons in ultrasound modulated optical tomography of thick biological tissues,” Opt. Express, 12(22), 5469-5474 (2004). 
[20] S. Sakadzic, and L. V. Wang, "High resolution ultrasound-modulated optical tomography in biological tissues," Opt. Lett., 29(23), 2770-2772 (2004).

[21] G. Rousseau, A. Blouin, and J.-P. Monchalin, "Ultrasound-modulated optical imaging using a high-power pulsed laser and a double-pass confocal Fabry-Perot interferometer,” Opt. Lett., 34(21), 3445-3447 (2009).

[22] Y. Li, H. Zhang, C. Kim et al., "Pulsed ultrasound-modulated optical tomography using spectral-hole burning as a narrowband spectral filter,” Appl. Phys. Lett., 93(1), 011111 (2008).

[23] H. Zhang, M. Sabooni, L. Rippe et al., "Slow light for deep tissue imaging with ultrasound modulation," Appl. Phys. Lett., 100, 131102 (2012).

[24] P. Lai, X. Xu, and L. V. Wang, "Ultrasound-modulated optical tomography at new depth," Journal of Biomedical Optics, 17(6), 066006 (2012).

[25] Y. Suzuki*, P. Lai*, X. Xu et al., "High-sensitivity ultrasound-modulated optical tomography with a photorefractive polymer," Opt. Lett., In press, (2013). (* indicate equally-contributed authors)

[26] F. J. Blonigen, A. Nieva, C. A. DiMarzio et al., "Computations of the acoustically induced phase shifts of optical paths in acoustophotonic imaging with photorefractive-based detection,” Appl. Opt., 44(18), 3735-3746 (2005).

[27] D. Dalecki, "Mechanical Bioeffects of ultrasound," Annual Reviews of Biomedical Engineering, 6, 229-248 (2004).

[28] [American National Standard for the Safe Use of Laser in Health Care Facilities, ANSI Z1361.1] American National Standards Institute, (2000).

[29] G. Rousseau, A. Blouin, and J.-P. Monchalin, "Ultrasound-modulated optical imaging using a powerful long pulse laser," Opt. Express, 16(17), 12577-12590 (2008).

[30] S. Farahi, G. Montemezzani, A. A. Grabar et al., "Photorefractive acousto-optic imaging in thick scattering media at $790 \mathrm{~nm}$ with a $\mathrm{Sn}_{2} \mathrm{P}_{2} \mathrm{~S}_{6}$ :Te crystal," Opt. Lett., 35(11), 1798-1800 (2010).

[31] L. Wang, and S. L. Jacques, "Use of a laser beam with an oblique angle of incidence to measure the reduced scattering coefficient of a turbid medium," Appl. Opt., 34(13), 2362-2366 (1995).

[32] T. Murray, P. Lai, and R. Roy, "Measuring Tissue Properties and Monitoring Therapeutic Responses Using Acousto-Optic Imaging,” Annals of Biomedical Engineering, 40(2), 474-485 (2012).

[33] M. Gross, M. Lesaffre, F. Ramaz et al., "Detection of the tagged or untagged photons in acousto-optic imaging of thick scattering media by photorefractive adaptive holography," Eur. Phys. J. E, 28(2), 173-182 (2009).

[34] J. Thomas, M. Eralp, S. Tay et al., "Photorefractive polymers with sub-millisecond response time," Proc. SPIE, 6335, (2006). 